\title{
THE ROLE OF PATIENTS AND MEDICAL SPECIALISTS IN ACHIEVING HIGHER LEVEL OF THERAPY COMPLIANCE- AN EXAMPLE WITH WILLINGNESS TO PARTICIPATE IN CLINICAL TRIALS
}

\author{
Svetoslav Stoev ${ }^{1}$, Vladimir Atanasov², Ilko Getov ${ }^{3}$
}

\begin{abstract}
BACKGROUND: Patients' compliance is a leading factor in achieving determined endpoints of a treatment process. Low patient compliance with prescribed therapeutic plan is very common in both ambulatory practice and clinical trials. Non-adherence of subjects in clinical studies can seriously jeopardize validity of results obtained during randomized clinical trials (RCTs).
\end{abstract}

METHODS: Both systematic literature review and cross-sectional survey were conducted among physicians and patients to determine evaluation of therapy adherence by both groups.

RESULTS: According to the analytical reviews in published articles, the most common definition of therapy adherence or compliance is "the ability and willingness of patients to abide to a prescribed therapeutic regimen". There is a strong correlation between patient adherence to the therapeutic regimen determined by the study protocol of RCTs, and the validity of efficacy and safety evaluation of the investigated medicinal product. Analysis of the results from the conducted survey lead to the conclusion that $35 \%$ of physician's state that the results of ongoing therapy are highly dependent on patient compliance. As far as patients are concerned, almost half of the respondents have answered they comply with the prescribed therapy and would evaluate the treatme nt process as successful. Both patients and medical professionals declare their willingness to participate in clinical trials and strictly stick to the requirements of the study protocol.

CONCLUSION: These results decisively confirmed that the control of a disease is a bilateral process between the patient and the doctor. Patient satisfaction with their current therapy directly correlates with the results of treatment. Majority of patients evaluate their possible participation in a clinical trial as an opportunity to get an access to a better medical service. Both medical professionals and patients declare their strong motivation to comply with the therapeutic plan if it is a part of a clinical study protocol.

UDC Classification: 615.4, DOI: http://dx.doi.org/10.12955/cbup.v4.847

Keywords: therapeutic compliance, PASS, PAES, therapy adherence, clinical trials.

\section{Introduction}

Therapeutic compliance can be defined as a patient willingness to strictly adhere to the therapeutic and diagnostic procedures determined by healthcare professionals involved in the treatment process. The outcome of the disease management plan depends in many cases on the level of patient cooperation while performing treatment procedures and implementation of prescribed recommendations for ambulatory recovering. Therapeutic compliance during participation in clinical trials is a complicated mixture of several factors like adherence to drug regimen, compliance with a specific prescribed life style or a diet, changing work schedules or even changing family habits. In referral to ambulatory practice, noncompliance can lead to non-recovery, and recurrence of the diagnosed illness or even life threatening situations. However, when a compliance deviation is met in clinical trials, consequences can be much direr and these deviations can lead to incorrect conclusions and implementation of bad treatment practices. In the process of compliance management in both ambulatory and clinical practices, the recognition and consequently prevention of factors that could lead to patient noncompliance must first be achieved.

\section{Methodology}

It was conducted as a systematic review of published scientific literature to analyze factors that affect the willingness of patients to comply with the prescribed treatment by doctors. For the purpose of literature analysis, a Google, PubMed and Scopus search was conducted using the keywords: "compliance", "clinical trials", "participation in clinical studies", "non-compliance", and "therapy

\footnotetext{
${ }^{1}$ Svetoslav Stoev, Pharmacy Faculty, Medical University Sofia, Bulgaria, slavi1702@ gmail.com

2 Vladimir Atanasov, Pharmacy Faculty, Medical University Sofia, Bulgaria, quest_atanasov@yahoo.com

${ }^{3}$ Ilko Getov, Pharmacy Faculty, Medical University Sofia, Bulgaria, ilko.getov@ gmail.com
} 
adherence/non-adherence". Only articles published between 2000 and 2015 were eligible for literature review.

A survey among 119 physicians and 117 patients was conducted to evaluate their willingness to participate in post-marketing RCTs. The response rate in both samples of respondents is $93 \%$. As far as the patient's interview is concerned the eligible criteria for respondents was: patients (both impatient and outpatient) who are 18 years or more of age, literate and have fully understood the purpose of the survey.

The inclusion criteria for medical doctors, included in the survey was:

- Senior doctors from both primary and secondary care, who have obtained diploma in at least one medical specialty and have been principle investigators or sub investigators in at least one clinical trial;

- Medical doctors - residents, who have finished at least their first year of education to obtain a medical specialty and have participated in the conduction of at least one clinical trial;

- The survey form has been completed only by doctors who are currently practicing in Republic of Bulgaria;

- The questionnaire was structured in accordance with the "funnel principle" - starting from more general questions and proceeding, to issues directly related to the main purposes of the study.

\section{Results and Discussion}

Review of the therapeutic compliance

The basis of a proper and significant discussion about therapeutic compliance is figuring out a clear and accurate definition of the term "compliance". Oxford dictionary defines compliance as "the practice of obeying rules or requests made by people in authority" (Oxford Advanced Learner's Dictionary of Current English; Stenson \& Hornby, 1996). Literature review of published articles on this topic revealed that for health care professionals, compliance matches the perspective of a term, describing the situation when "patient's behaviors (in terms of taking medication, following diets, or executing life style changes) coincide with healthcare providers' recommendations for health and medical advice" (Jin, Sklar, Min Sen Oh, \& Chuen Li, 2008). Other authors propose almost similar interpretations, coded by terms "adherence" or "concordance", which are absolutely interchangeable and a describe patient's "ability and willingness to abide by a prescribed therapeutic regimen" (Jin et al., 2008). The majority of articles that focus on patient-prescriber relationship emphasize that each of the word formations used for naming compliance, stress on the leading role and responsibility of patient for strict implementation of therapeutic regimen. On this basis, non-compliance is defined by most of the authors as a situation "when a person, being an object of treatment does not agree at the needed stage with the prescribed therapy, disease management plan or recommendations of the healthcare professionals" (Besch, 1995). The spectrum of compliance includes not only the therapeutic treatment, but adherence to all the prescribed diagnostic procedures, treatment interventions, advices on lifestyle and preventive actions, and follow up activities (Besch, 1995). In case the main focus of patient adherence is limited to the medication therapy, one of the most objective ways to measure compliance is retrospective comparison of actual dosing times-series versus originally prescribed therapeutic scheme and the extent of correspondence between them (Besch, 1995).

Definition of compliance, discussed above, casts a doubt regarding the accuracy of different compliance measurement methods. Different authors propose several methods as gold standard for measuring the stage of correspondence between what has been taken by the patient versus what has been prescribed by the physician. There are several factors that can vitiate accurate measurement of a patient's compliance. These factors can be identified in two separate groups: patient-related factors, and factors related to shortcomings of registers and/or evaluation methods. These two types of biases influence the final evaluation of patient adherence in different stages. The section on patient-related factors includes biases like manipulation of dosing logs, non-refilling of prescriptions or deviations in duration of periods between dispensations, lack of memory for date of dispensing, or not taking pills at all. Concerns in referral with design of adherence evaluation methods are connected with deviations in patient register maintenance, filling wrong dates of dispensing or disadvantages of drug monitoring 
procedures like the necessity of taking blood samples, performing difficult assays, or inclusion of chemical markers of drug use like digoxin, in therapeutic schemes (Farmer, 1999). All these shortcomings are not only hazardous for the accuracy of compliance evaluation but can put patients at risk (Pullar, Kumar \& Feely, 1989; Haynes \& Dantes, 1987; Farmer, 1999).

While non-compliance in general ambulatory practice can lead to undesired health outcomes for a certain patient, therapy non-adherence during controlled randomized clinical trials may put the validity of study results at risk and lead to wrong interpretations in routine clinical practice. Non-adherence in clinical trials may result in misleading conclusions and influence the therapeutic tendencies and guidelines which indirectly lead to consequences for the public health. The situation with compliance management in clinical trials is much more specific, not only because of the sophisticated protocols and specific inclusion and exclusion criteria, but also because of the different objectives of each clinical research project (Haynes \& Dantes, 1987). Furthermore, patient compliance in clinical trials is strongly connected with the study design and purpose. For example, in cases where trial objectives include evaluation of efficacy, screening of participants should prevent non-compliant subjects to be recruited. However, when the study scope is estimation of effectiveness the situation is just the opposite (Farmer, 1999; Haynes \& Dantes, 1987). At first sight, compliance in clinical trials seems to be guaranteed by the precise and permanent monitoring of subjects enrolled in the clinical trials, but in practice, the final analysis and report of trial results should contain evaluation of deviations, caused by low subject compliance and the percentage of participants who have not adhered to the approved regimen and following consequences. In addition, an effective strategy for improvement of compliance should be provided when study protocol is being developed (Farmer, 1999).

The methods for compliance measuring in clinical trials is almost the same as adherence evaluation techniques used in ambulatory care i.e. direct methods including analysis of drug levels in blood and urine, usage of chemical markers, and controlled dispensation of investigational products. Indirect algorithms include interview of patients regarding medication usage, completion of dosing logs, counting of tablets or used vials and monitoring by electronic logs. Shortcomings in these cases are related to difficult detection of non-compliance, impossible or postponed detection of consequences of non-adherence, and measurement of the extent of non-adherence, especially when large cohorts of patients are involved in multicenter randomized clinical trials. Although clinical trials are strictly regulated field, numerous hi-tech methods are implemented in their conduction. There are many modern approaches like electronic Medication Event Monitoring System (MEMS) that occur to be promising methods for tracing subject compliance level. MEMS successfully replaces archaic methods like pill counts and meets the approved criteria for validity and sensitivity of compliance measures. Namely, validity and reliability of adherence measuring approaches should be the leading factor in the researcher's decision regarding what compliance improving instruments and techniques to use (Farmer, 1999).

According to Besch (1995), improving adherence to treatment regimen in clinical trials is not only the responsibility of clinical researcher but also the trial subjects who should be aware of the fact that their behavior directly influences trial results. Patient responsibility is not limited only to taking medication on time and in right dosage. Participants should stick to strict schedules for visits, follow up monitoring meetings, follow advices regarding adherence to a diet or a specific lifestyle or even change their working hours in accordance with the treatment regimen. Avoidance of compliance deviations is guaranteed by a complex compilation of all these factors (Besch, 1995; Haynes \& Dantes, 1987; Jin et al., 2008). According to most of the published articles poor subject compliance can seriously reduce the power of clinical trial results. Researchers have calculated that if roughly one third of patients, recruited for a clinical study are non-compliers, the number of participants in the trial must be doubled so that the validity and generality of results to be proved is maintained (Pullar, Kumar \& Feely, 1989). The risk of reporting non-valid results or wrong conclusions resulting in misleading interpretation of results from randomized trials is even bigger when non-compliers participate in comparative studies. For example, when a study compares two products that should be taken in the same way, and one of these products is being taken by a subject with poor compliance, comparison of concentrations circulating in blood will be jeopardized. The situation may need even more sophistication when the investigated product is long-acting or the study purpose is safety evaluation of compared drugs. In these situations, traditional methods used for measuring therapeutic 
compliance are not always accurate and sometimes can even overestimate compliance and prove results which are not valid and cannot act as a ground for clinically correct conclusions (Pullar, Kumar \& Feely, 1989).

Results from survey among physicians and patients

One of the aims of the survey conducted among medical professionals was to evaluate doctors' attitude towards the treatment of their patients.

The obtained results, are represented graphically in Figure1.

Figure 1: Distribution of answers, provided by doctors, being asked to determine whether ischemic heart disease of their patients is well controlled.
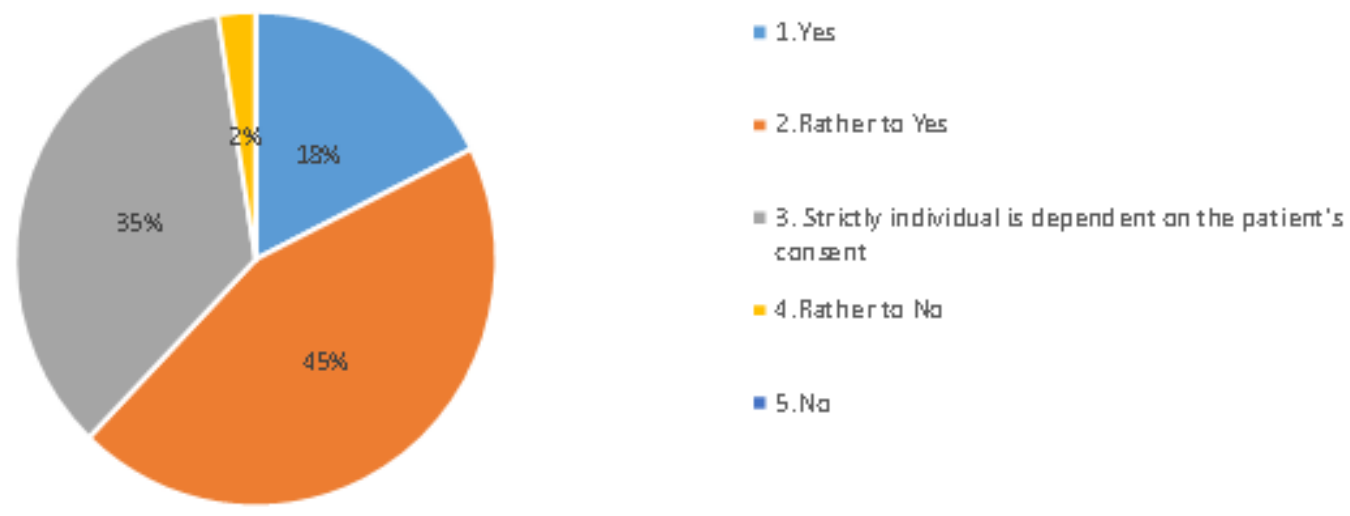

Source: Authors

The analysis of results clearly showed that over $50 \%$ of doctors who participated in the survey believe that ischemic diseases for their patients are well controlled, 35\% of physicians stated that the results of ongoing therapy are highly dependent on patient compliance, and only $2 \%$ of patients responded that their disease is not well controlled.

These results confirm decisively that the control of a disease is a bilateral process between patient and doctor. Experts are required to diagnose timely and accurately, to identify an effective and precise therapy plan. No less important stage of treatment are provided as clear recommendations or advices to patients during the therapeutic course. On the other hand the effective therapeutic results are not less dependent on the level of patient compliance with prescribed treatment.

Although pharmacists are not subject to the current study, they occupy an extremely important part in the treatment of patients. They have the function of a mediator in the process of prescribing and administration of medicines. When these health professionals become an integral part of the treatment process, the results from the prior consent of patients will increase. Satisfactory effects for patients and consequently, for the public would be achieved as a result of these joint efforts.

In assessing the satisfaction of surveyed patients from their current therapy it was clearly observed that there is a dichotomy in their attitude and evaluations of the therapeutic regimen. Patient satisfaction with their current therapy directly correlates with the results of treatment. It expresses the degree of involvement in the execution of their daily habits, but also from the mental and physical state that directly connects with the results of their therapy.

The analysis of the results shows that $9 \%$ of the patients cannot assess their implementation therapy. It could also be a problem due to poor communication with doctors, low accordance with prescribed drug therapy and the recommendations provided by medical specialists.

When directly asked about the willingness of patients to participate in a clinical study, respondents said emphatically positive attitude towards their possible participation. Data obtained by asking this question can be seen in Figure 3 . 


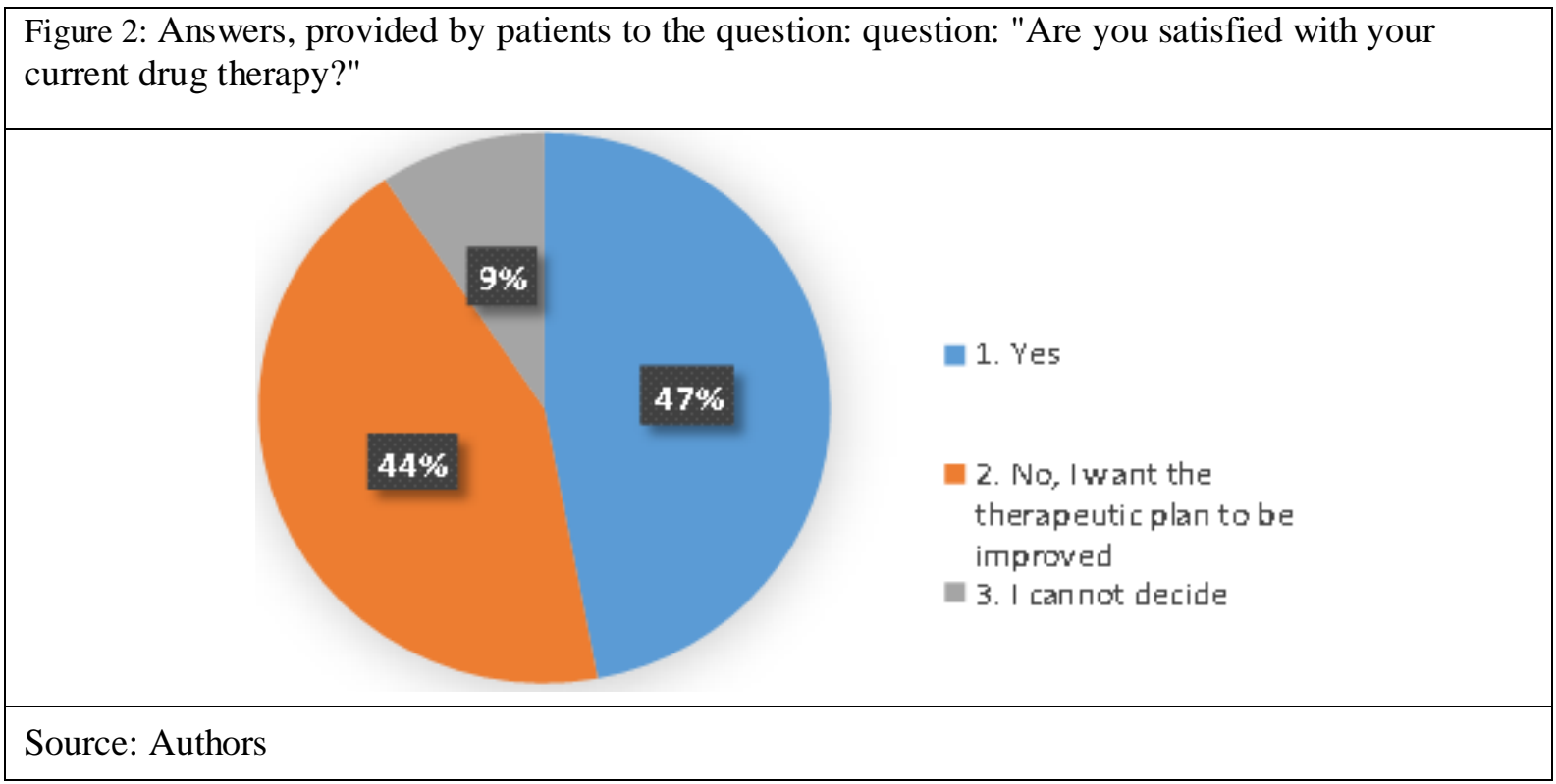

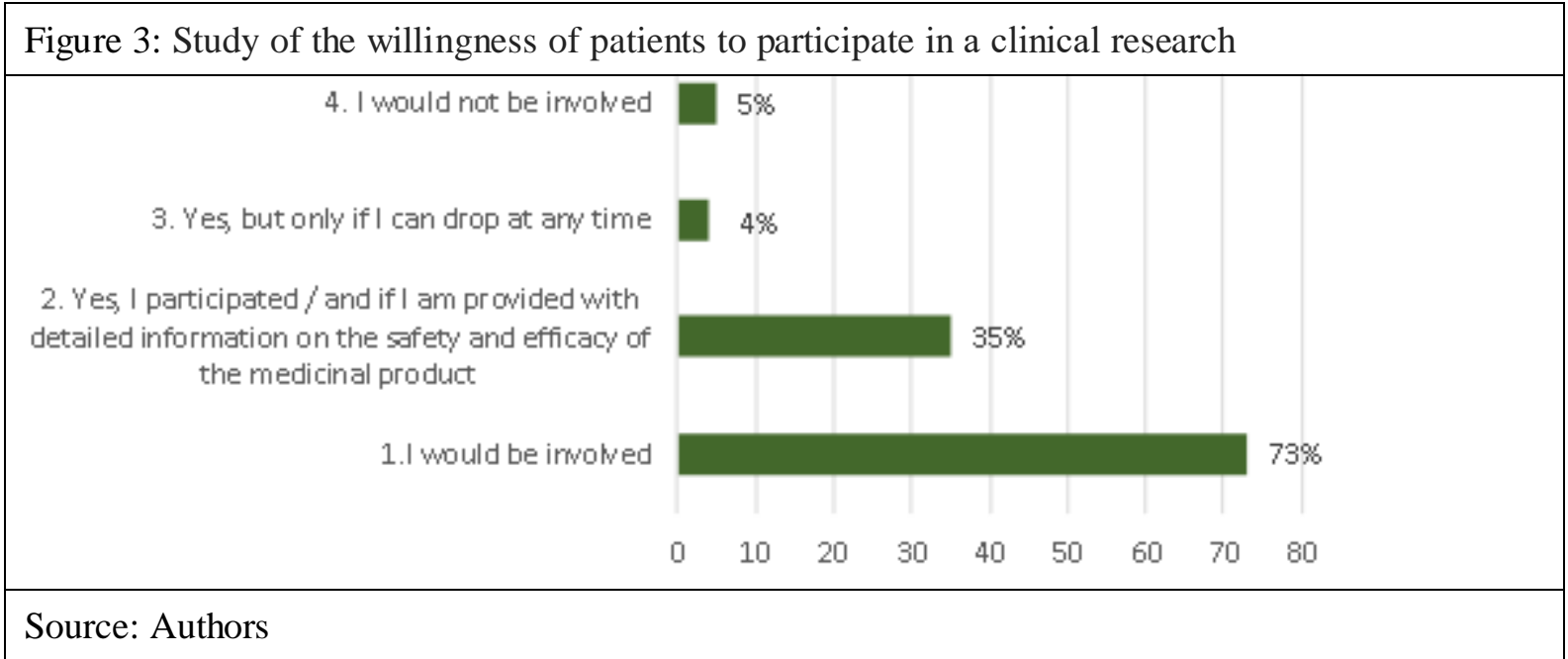

The majority of respondents stated that they would participate in a clinical study to get a chance to improve their therapy. Some of them would trust the process, after being provided additional information about the investigated therapy. The results strongly suggest that the surveyed patients declare their willingness to participate in clinical studies, so that they can contribute to the process of gathering/obtaining additional data on the efficacy and safety of a specific medicinal product. Patients find their participation in a clinical trial an opportunity to get a better chance to improve their current treatment and access an effective therapeutic scheme. They also believe that being involved in a clinical research project, they will receive medical care, painstaking monitoring of the disease and consultation with leading medical professionals.

Another aim of the survey was to investigate the patients' willingness to perform their duties according the Protocol of the clinical trial and their willingness to stick to the provided requirements. The analysis of the questionnaire results outlined that patients declare their conscious intention to follow strictly the procedures according to the investigational plan.

Compliance with the procedures, defined by the Study plan can be traced by researchers and clinical monitors during the preparation and conduct of the study. The abuse of procedures and deviations from protocol of the study is a major problem while conducting clinical trials and would seriously invalidate the results, vitiating the possibility of proving that the product meets the safety and efficacy endpoints. 


\section{Conclusion}

Both patients and medical professionals declare their conscious willingness to participate in the process. However, the majority of patients are not aware of the specific characteristics and limitations of post-marketing studies and are not able to differentiate interventional trials from non-interventional trials. As far as physicians are concerned, they are well trained and demonstrate high grade of awareness of conducting phase IV clinical trials, both interventional and non-interventional, including design, methodology and endpoints of the study.

Regarding the attitude to the therapy prescribed, results from both patients and medical professional groups lead to the conclusion that the positive evaluation of the therapeutic plan prevails. However, what features the two investigated cohorts may leave scope for frustration from the therapy. Majority of patients state the lack of effectiveness of treatment as the main reason for their negative attitude to the therapeutic approach, while most of the doctors explain the poor improvement in the condition of their patients by the patients' non-compliance and failure in following the prescriber`s advice.

\section{References}

Besch, C. L. (1995). Compliance in clinical trials. Aids, 9(1), 1-10. DOI:10.1097/00002030-199501000-00001

Farmer, K. C. (1999). Methods for measuring and monitoring medication regimen adherence in clinical trials and clinical practice. Clinical Therapeutics, 21(6), 1074-1090. DOI:10.1016/s0149-2918(99)80026-5

Haynes, R., \& Dantes, R. (1987). Patient compliance and the conduct and interpretation of therapeutic trials. Controlled Clinical Trials, 8(1), 12-19. DOI:10.1016/0197-2456(87)90021-3

Jin, J., Sklar, G. E., Min Sen Oh, V., \& Chuen Li, S. (2008). Factors affecting therapeutic compliance: A review from the patient's perspective. Therapeutics and Clinical Risk Management, 4(1), 269-286.

Pullar, T., Kumar, S., \& Feely, M. (1989). Compliance in clinical trials. Annals of the Rheumatic Diseases, 48(10), 871-875. DOI:10.1136/ard.48.10.871

Stenson, N., \& Hornby, A. S. (1996). Oxford Advanced Learner's Dictionary of Current English. The Modern Language Journal, 80(3), 412. DOI:10.2307/329464 